\title{
Long-Term Relationship between Atrial Fibrillation, Multimorbidity and Oral Anticoagulant Drugs Use
}

\author{
Marco Proietti ${ }^{1,2,3,4 *} \mathrm{MD}$, Irene Marzona ${ }^{1 *} \mathrm{MSc}$, Tommaso Vannini ${ }^{1} \mathrm{MSc}$, \\ Mauro Tettamanti ${ }^{1} \mathrm{MSc}$, Ida Fortino ${ }^{5} \mathrm{MD}$, Luca Merlino ${ }^{5} \mathrm{MD}$, Stefania Basili ${ }^{6} \mathrm{MD}$, \\ Pier Mannuccio Mannucci ${ }^{5}$ MD, Giuseppe Boriani ${ }^{6}$ MD PhD, \\ Gregory YH Lip ${ }^{2,7,8}$ MD, Maria Carla Roncaglioni ${ }^{1}$ MSc, Alessandro Nobili1 MD
}

${ }^{1}$ Istituto di Ricerche Farmacologiche Mario Negri IRCCS, Milan, Italy; ${ }^{2}$ Liverpool Centre for Cardiovascular Science, University of Liverpool, Liverpool, United Kingdom; ${ }^{3}$ Department of Clinical Sciences and Community Health, University of Milan, Milan, Italy; ${ }^{4}$ Geriatric Unit, Fondazione IRCCS Ca' Granda Ospedale Maggiore Policlinico, Milan, Italy; ${ }^{5}$ Regional Ministry of Health, Lombardy Region, Milan, Italy; ${ }^{6}$ Department of Translational and Precision Medicine, Sapienza-University of Rome, Rome, Italy; ${ }^{7}$ Scientific Direction, Foundation IRCCS Ca' Granda Ospedale Maggiore Policlinico, Milan, Italy; ${ }^{8}$ Department of Biomedical, Metabolic and Neural Sciences, University of Modena and Reggio Emilia, Policlinico di Modena, Modena, Italy; ' ${ }^{2}$ Liverpool Centre for Cardiovascular Science, University of Liverpool and Liverpool Heart \& Chest Hospital, Liverpool, UK; ${ }^{10}$ Aalborg Thrombosis Research Unit, Department of Clinical Medicine, Aalborg University, Aalborg, Denmark.

*Both authors equally contributed to this manuscript.

Data from this manuscript have been partially previously presented as an abstract in the 2018 European Society of Cardiology Meeting, $25^{\text {th }}-29^{\text {th }}$ August 2018, Munich, Germany. 
Manuscript Word Count: 3210

Tables: 3

Figures: 2

References: 36

\section{FUNDING}

No funding was provided for this project.

\section{DISCLOSURES}

MP reports consultant activity for Boehringer Ingelheim. PMM reports honoraria for lectures as speaker or chair symposia organized by Bayer, Grifols, Kedrion, LFB, Novo Nordisk and Pfizer; scientific consultant for Bayer, Baxalta and Kedrion. GB reports small speaker's fee from Medtronic, Boston, Boehringer and Bayer. GYHL reports consultant activity for Bayer/Janssen, BMS/Pfizer, Biotronik, Medtronic, Boehringer Ingelheim, Microlife and Daiichi-Sankyo. Speaker activity for Bayer, BMS/Pfizer, Medtronic, Boehringer Ingelheim, Microlife, Roche and Daiichi-Sankyo. No fees are received personally. All relationship disclosed are related to activities performed outside the submitted work. All other authors have no disclosures to declare.

\section{ACKNOWLEDGMENTS}

We thank Igor Monti from Istituto di Ricerche Farmacologiche Mario Negri IRCCS, Simone Schiatti and Giovanna Rigotti from Lombardia Informatica S.p.A, Alfredo Bevilacqua from SANTER Reply S.p.A.

Corresponding Author

Dr. Marco Proietti 
IRCCS - Istituto di Ricerche Farmacologiche "Mario Negri"

Via Giuseppe La Masa 19, 20156, Milan, Italy

e-mail: marco.proietti@uniroma1.it 


\section{ABSTRACT}

Objectives: To analyse the relationship between AF and Charlson Comorbidity Index (CCl) in a population-based cohort study over a long-term follow-up period, in relation to oral anticoagulant (OAC) prescriptions and outcomes.

Patients and Methods: We used data from the administrative health databases of Lombardy. All AF patients $\geq 40$ years admitted to hospital in 2002 were considered for analysis and followed up to 2014 . AF diagnosis and CCl were established according to ICD-9 codes.

Results: In 2002, 24,040 patients were admitted with a diagnosis of AF. CCI was higher in AF patients compared to non-AF cases (1.8 \pm 2.1 vs. $0.2 \pm 0.9, P<.001)$. Over 12-years of follow-up, AF was associated with an increased risk of higher $\mathrm{CCl}$ (beta coefficient: 1.69, 95\% confidence interval [Cl]: 1.67-1.70). In AF patients, $\mathrm{CCl}$ was inversely associated with OAC prescription at baseline $(P<.001)$ and at the end of the follow-up $(P=.03)$. AF patients with a high $\mathrm{CCl}(\geq 4)$ had a higher cumulative incidence for stroke, major bleeding and allcause death (all $\mathrm{P}<.001$ ), compared to those with low $\mathrm{CCl}(0-3)$. Adjusted Cox regression analysis found that time-dependent continuous $\mathrm{CCl}$ was associated with an increased risk for stroke, major bleeding and all-cause death (all $\mathrm{P}<.001$ ).

Conclusions: In hospitalized patients, $\mathrm{AF}$ is associated with an increase in $\mathrm{CCl}$, that was inversely associated with $\mathrm{OAC}$ prescriptions during follow-up. $\mathrm{CCl}$ is independently associated with an increased risk of stroke, major bleeding and all-cause death.

KEYWORDS: atrial fibrillation; multimorbidity; oral anticoagulant drugs; outcomes. 


\section{INTRODUCTION}

Atrial fibrillation (AF) has an increasing incidence, prevalence and impact on healthcare systems globally ${ }^{1}$. The worldwide AF epidemic is mainly attributed to an increasing ageing population ${ }^{2}$. AF patients are often older and more affected with concomitant cardiovascular (CV) and non-cardiovascular conditions, that affect significantly patients' clinical course, leading to an increased risk of $\mathrm{CV}$ and all-cause death ${ }^{3}$.

The concept of multimorbidity (defined as the concomitant presence of two or more chronic conditions) has gained much medical attention in the last decades ${ }^{4}$. As with AF, the prevalence of multimorbidity increases with increasing age and is associated with a high risk of mortality, reduced functional status, increased healthcare expenditure and use of resources ${ }^{5}$. As part of the biological, sociological and clinical complexity associated with healthcare ${ }^{6}$, multimorbidity demands solid integrated care and a holistic approach to the patient in order to properly manage the associated risks ${ }^{5}$. Moreover, multimorbidity is very common in patients with CV disease ${ }^{7}$.

The Charlson Comorbidity Index $(\mathrm{CCl})$ has been validated as a reliable tool to evaluate the burden of multimorbidity in the general population and is significantly associated with an increased risk of all-cause death during the long-term follow-up ${ }^{8}$. Furthermore, $\mathrm{CCl}$ has been extensively validated in patients with $\mathrm{CV}$ disease $^{9}$. Nevertheless, despite $\mathrm{AF}$ being associated with several comorbidities ${ }^{1}$, scarce data exist about the overall burden of multimorbidity and the relationship of $\mathrm{CCl}$ with $\mathrm{AF}$.

The aim of this report is to evaluate the relationship between AF, burden of multimorbidity (as defined by $\mathrm{CCl}$ ), the prescription of oral anticoagulant (OAC) drugs and long-term 
outcomes in a large population-based cohort of AF patients from Lombardy, the largest region of Northern Italy.

\section{METHODS}

\section{Data Source and Study Population}

This study used linkable administrative health databases of the Lombardy Region which include the demographic data of all residents and detailed information on hospital admissions and drug prescriptions. To date, with a population of more than 10 million inhabitants, Lombardy is the largest Italian region, comprising highly populated urban areas, as well as industrial and rural ones. The Italian healthcare system is based on a public National Health Service, which provides free assistance to anyone on the National territory, irrespective of any social, economic and clinical pre-existing condition. A personal identification code is given to each subject and kept in the National Civil Registration System.

All databases are linked anonymously using unique encrypted patient codes, in accordance with the Italian privacy regulations. By virtue of a specific agreement between the Mario Negri Institute and the Lombardy Region, for the use of the anonymous administrative data derived from these databases it was not necessary to obtain approval from any ethics committee. Data were available for fifteen consecutive years, from 2000 to 2014. For any hospital admission, all discharge diagnoses have been coded according to International Classification of Disease $9^{\text {th }}$ revision [ICD-9]. Moreover, the hospital discharge database records the date of hospital admission, date of discharge or death and procedures performed during admission. The drug prescription database contains the drug name and its Anatomical Therapeutic Chemical classification code, quantity and dispensation date after the discharge at home, but not during the index hospitalization. All 
data about subjects $\geq 40$ years old ( $>6$ million inhabitants) were available and included in this analysis.

Data from 2000 to 2001 were used to build the clinical history of patients and to calculate baseline $\mathrm{CCl}$. Year 2002 was used as index year to evaluate AF diagnosis. All discharge diagnoses were searched for codes 427.31 and 427.32 , and all subjects with these codes irrespective of position, were assigned to the group of patients with prevalent AF. A random sample of non-AF patients ten times greater than those with AF was taken as a control group.

\section{Definition of Concomitant Conditions and CCl Calculation}

According to the diagnoses reported at discharge and coded as per ICD-9, all patients were evaluated for presence of concomitant conditions (see Supplementary Materials, eTable 1). Hypertension was identified on the basis of prescription of at least one antihypertensive drug in the six months after entering the study cohort (see eTable 1 for ATC codes). Accordingly, the $\mathrm{CHA}_{2} \mathrm{DS}_{2}$-VASc score was computed according to the original definitions ${ }^{10}$. A modified version of HAS-BLED (mHAS-BLED), calculated excluding the 'L' criterion about quality of oral anticoagulation control, was computed according to previous studies ${ }^{11}$.

In its original definition, $\mathrm{CCl}$ comprised 19 diagnosis to which different weights have been assigned and summed to obtain the final calculation of $\mathrm{CCl}^{8}$ (eTable 2). For this study, the $\mathrm{CCl}$ was calculated according to a validated method applied to the administrative databases $^{12}$. All AF patients were grouped according to $\mathrm{CCl}$ as patients with low multimorbidity $(\mathrm{CCl} 0-3)$ and a high multimorbidity $(\mathrm{CCl} \geq 4)$. 
In order to analyse the relationship between $\mathrm{AF}$ and $\mathrm{CCl}$, we analyzed differences at baseline between AF and non-AF patients. Then, we analyzed the relationship between $\mathrm{AF}$ and $\mathrm{CCl}$ throughout the follow-up observation, to establish whether or not a significant association exists between AF and increasing multimorbidity burden according to $\mathrm{CCl}$.

\section{Oral Anticoagulant Drugs}

In the purpose of the study we evaluated OAC prescription at baseline and at the end of observation according to $\mathrm{CCl}$. At the beginning of observation, only vitamin $\mathrm{K}$ antagonists (VKAs) were available, while at the end of observation the non-vitamin $\mathrm{K}$ antagonist oral anticoagulants (NOACs) became available for prescription. OAC drugs prescription were recorded as follows: VKA (warfarin: B01AA03, acenocumarol: B01AA07); NOACs (dabigatran: B01AE07, rivaroxaban: B01AF01, apixaban: B01AF02, edoxaban: B01AF03), antiplatelet drugs (B01AC). Additionally, we evaluated discontinuation of OAC throughout the study period and the impact of the burden of multimorbidity in determining the occurrence of OAC discontinuation.

\section{Study Outcomes}

Outcomes of interest for the present study were: stroke, major bleeding and all-cause death (see Supplementary Materials, Table S1 for ICD-9 codes). Follow-up observation started when the patient entered the study cohort and proceeded until one of the outcomes occurred or when the follow-up was censored. Reasons to be censored included emigration, admission to a nursing home, occurrence of death or reaching the end of the follow-up observation.

\section{Statistical Analysis}


Continuous variables, expressed as mean and standard deviation, were compared across the groups using Student's t-test. Categorical variables, expressed as counts and percentages, were compared using Chi-square test.

To analyse the relationship between $\mathrm{AF}$ and $\mathrm{CCl}$ over the follow-up period, we performed a mixed linear effect logistic model adjusted for years of observation, age, sex and an interaction term between AF and follow-up years. A supplementary age-stratified analysis ( $<65$ years, $65-74$ years, $\geq 75$ years) was also performed.

To evaluate the impact of $\mathrm{CCl}$ in $\mathrm{OAC}$ prescription for $\mathrm{AF}$ patients, we performed a logistic regression model, adjusted for age and sex, for OAC prescription at baseline and at the end of the follow-up. $\mathrm{CCl}$ was considered as a continuous variable and as classes (high vs. low multimorbidity). At the end of follow-up, we also evaluated separately prescription of VKA and NOACs. Impact of $\mathrm{CCl}$ in determining OAC discontinuation was evaluated with a competitive risk model adjusted for concomitant risk of all-cause death and adjusted with age and sex.

Differences in survival between AF patients with low and high multimorbidity were analyzed with Log-Rank test and Kaplan-Meier curves were drafted accordingly. A Cox regression analysis to evaluate the impact of $\mathrm{CCl}$, considered as a continuous timedependent variable, in determining study outcomes was performed. Two Cox regression models were performed, as follows: i) adjusted for age and sex; ii) adjusted for age, sex and OAC prescription. A two-sided value of $p \leq 0.05$ was considered statistically significant. Analyses were performed with Stata 13.0 (Stata Corp LP, College Station, TX, USA), and SAS software, version 9.4 (SAS Institute). 


\section{RESULTS}

In 2002, a total of $24,040 \mathrm{AF}$ patients were retrieved, as well as 240,400 non-AF patients. At baseline (Table 1), AF patients had a significantly higher mean $( \pm S D) \mathrm{CCl}$ than non-AF subjects $(1.8 \pm 2.1$ vs. $0.2 \pm 0.9, P<.001)$. Patients with $A F$ were significantly older and more likely male, and more likely affected by comorbidities compared to non-AF subjects. Accordingly, AF patients had a significantly higher mean $( \pm S D) \mathrm{CHA}_{2} \mathrm{DS}_{2}$-VASc score (3.3 \pm 1.4 vs. $1.4 \pm 1.2, \mathrm{P}<.001)$ and mHAS-BLED $(2.3 \pm 1.0$ vs. $0.9 \pm 1.0, \mathrm{P}<.001)$ compared to non-AF subjects.

Within the overall AF patient cohort, 4,295 patients (17.9\%) had high multimorbidity (CCl

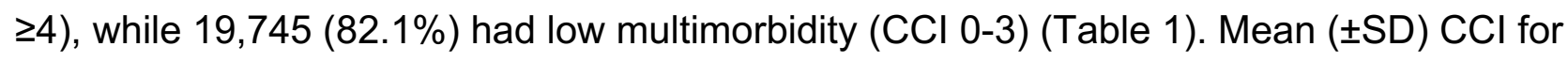
the high multimorbidity group was $5.5 \pm 1.8$, while for the low multimorbidity group, $1.1 \pm 1.1$ $(P<.001)$. Patients with high multimorbidity were older and more likely male than those with low multimorbidity (both $\mathrm{P}<.001$ ). In patients with high multimorbidity all conditions considered were more prevalent, except for hypertension which was more prevalent in the low multimorbidity group $(P<.001)$. Patients with high multimorbidity had a higher thromboembolic risk than the low multimorbidity group (mean $[ \pm \mathrm{SD}] \mathrm{CHA}_{2} \mathrm{DS}_{2}-\mathrm{VASc}$ score $4.1 \pm 1.5$ vs. $3.2 \pm 1.3$, respectively; $P<.001$ ). Similarly, patients with high multimorbidity had a higher baseline bleeding risk than those with low multimorbidity (mean [ \pm SD] mHASBLED score $2.8 \pm 1.1$ vs. $2.1 \pm 0.9$, respectively; $P<0.001$ ). At baseline, patients with high multimorbidity were significantly less prescribed with OAC than those with low multimorbidity $(30.0 \%$ vs. $42.3 \%, P<.001)$.

\section{Trends in $\mathrm{CCl}$ and Relationship with $\mathrm{AF}$} A mixed linear effect logistic model was compiled to analyse the relationship between AF and $\mathrm{CCl}$. Overall, $\mathrm{CCl}$ progressively increased over time both in non-AF and $\mathrm{AF}$ patients, 
being increasingly and steadily higher in AF patients compared to non-AF ones $(P<.001)$

[Figure 1]. After adjustment for years of observation, age, sex and an interaction term between $\mathrm{AF}$ and years of observation, $\mathrm{AF}$ was associated with a progressively higher $\mathrm{CCl}$ (beta coefficient: 1.69, 95\% confidence interval [Cl]: 1.67-1.70), $F=99943.8, P<.001)$. Further, the interaction term between AF and years of observation was also independently associated to the progressively higher $\mathrm{CCl}(\mathrm{P}<.001)$. Subgroup analysis for age classes, showed that this relationship was consistently statistically significant for patients $<65$ years, 65-74 years and $\geq 75$ years (all $P<.001$ ) [eFigures $1-3$ ].

\section{$\mathrm{CCl}$ and OAC Prescription}

After adjustment for age and sex (Table 2), $\mathrm{CCl}$ as a continuous variable was inversely associated with OAC prescription (odds ratio [OR]: 0.91, 95\% Cl: 0.89-0.92). The high multimorbidity category $(\mathrm{CCl} \geq 4)$ was inversely associated with OAC prescription (OR: $0.65,95 \% \mathrm{Cl}: 0.60-0.70)$.

At the end of follow-up, even though $\mathrm{CCl}$ as a continuous variable was inversely associated with OAC prescription (OR: $0.98,95 \% \mathrm{Cl}: 0.98-0.99)$, the high multimorbidity category was not significantly associated (OR: $0.98,95 \% \mathrm{Cl}: 0.93-1.04)$. Examining separately VKA and NOACs prescription (Table 2), while there was no difference in VKA prescription, both continuous and categorical $\mathrm{CCl}$ were inversely associated with prescription of NOACs (both $\mathrm{P}<.001)$.

\section{Discontinuation of OAC}

Throughout the entire follow-up time, among the 9,646 patients prescribed at baseline with an OAC a total of 4,450 (46.1\%) discontinued their treatment. Median [IQR] time for discontinuation was 3 [1-7] years. In a competitive risk model (adjusted for the competitive 
risk of death), we verified that increasing $\mathrm{CCl}$ (as a time-varying independent variable) does not have any impact on risk of discontinuation (hazard ratio [HR]: $1.00,95 \% \mathrm{Cl}: 0.98-$ 1.01, $P=.61$ ), after adjustment for age and sex.

\section{Survival and Regression Analysis}

At follow-up, all the outcomes considered were more likely in the high multimorbidity group (eTable 3). Kaplan-Meier analysis shows that risk for stroke, major bleeding and all-cause death was consistently higher in high multimorbidity group compared to the low multimorbidity group [Figure 2].

Cox regression analysis (Table 3 ), using $\mathrm{CCl}$ as a continuous time-dependent variable to take account of the temporal increase and adjusted for age, sex and use of OAC, $\mathrm{CCI}$ was significantly associated with an increased risk for stroke (HR: 1.04, 95\% Cl: 1.02-1.06 per increasing point), major bleeding (HR: $1.03,95 \% \mathrm{Cl}$ : 1.01-1.06 per increasing point) and all-cause death (HR: 1.10, 95\% Cl: 1.09-1.11 per increasing point).

\section{DISCUSSION}

Our study showed that AF patients are exposed to a higher burden of overall multimorbidity than non-AF individuals, showing for the first time that a direct relationship exist between AF and an increasing multimorbidity burden over long-term follow-up, irrespective of age. Second, an increased burden of multimorbidity is inversely associated with OAC prescription, which could significantly affect AF patients' clinical history, despite not influencing treatment discontinuation. Third, an increased burden of multimorbidity in AF patients is directly and independently associated with an increased risk for stroke, major bleeding and all-cause death. 
The independent relationship between various single diseases and AF has been largely demonstrated. Indeed, several conditions contribute independently to incident AF occurrence and it has been suggested that tight control of concomitant risk factors and comorbidities could significantly reduce the burden of $A F^{13,14}$. Furthermore, several diseases are independently prevalent in AF patients ${ }^{15,16}$. Our paper first establishes a direct link between the presence of AF and the development of a progressively higher burden of multimorbidity. The evidence presented allows us to speculate about the role of AF as a proxy of a worse clinical status.

Our data are strengthened by the use of a solid and validated tool to evaluate multimorbidity such as the $\mathrm{CCl}$. Thus far, data about $\mathrm{CCl}$ in the contest of $\mathrm{AF}$ are scarce ${ }^{17}$. In a Belgian study derived from a primary care registry, a modified version of the $\mathrm{CCl}$ was found higher in AF elderly ( $\geq 60$ years) patients than in non-AF ones, also being associated with AF diagnosis ${ }^{17}$. The data presented in this study extend this previous evidence, confirming how the burden of multimorbidity is significant in AF patients, irrespective of age and of what may be single medical conditions. In particular, we show how a significant proportion of patients $(\sim 20 \%)$ had a high level of multimorbidity. A recent study derived from the UK Biobank, in a cohort of patients with self-reported AF which examined the presence of multimorbidity as the additive presence of various conditions, only $19.6 \%$ of patients reported no comorbidities and $11.1 \%$ of patients reported 4 or more comorbidities $^{18}$.

Our results show that increased multimorbidity in AF patients is significantly inversely associated with OAC prescription. This is a concerning trend, considering the associated increased thromboembolic risk. In the study by Vanbeselaere and colleagues, there was a possible inverse relationship between increasing $\mathrm{CCl}$ and reduced OAC prescription ${ }^{17}$. Our 
study extends previous knowledge, showing how this inverse relationship is consistent in a general population and over a long-term observation period. Moreover, we showed that if physicians appear to be more confident in prescribing VKA, the prescription of NOACs is significantly reduced in patients with increased multimorbidity. Our results substantiate previous observations that seem to suggest that AF patients prescribed with NOACs are relatively healthier and have less prevalent comorbidities (i.e. coronary artery disease, heart failure, pulmonary arterial hypertension, prior stroke) and were likely admitted with AF as main diagnosis ${ }^{15,19}$. Notwithstanding, since the study period included only the initial years of NOACs use, this could have influenced the choice of VKA rather than NOACs. Also, we cannot exclude that the physiological progressive worsening of renal function did influence the preferential choice of VKA in these patients.

In recent years, the increased risk of CV-related and all-cause death in AF cohorts has shifted the main focus of prevention of adverse events from stroke to mortality ${ }^{20-25}$. Our data show that the increased and increasing multimorbidity burden is strongly associated with an increased risk of all the adverse events, stroke, major bleeding and all-cause death. The Framingham Heart Study previously showed that AF patients with comorbidities have a consistently increased risk for cardiovascular events and all-cause death compared to those without ${ }^{26}$. An analysis from the "Outcomes Registry for Better Informed Treatment of Atrial Fibrillation" study showed that when AF patients were clustered according to the more frequent clinical characteristics, those in the 'lowcomorbidity' cluster had the lowest risk major cardiovascular and neurological adverse events than all the other identified clusters, variously affected by risk factors and other comorbidities $^{16}$. 
Our report also extends previous knowledge about the usefulness of $\mathrm{CCl}$ in AF patients. Thus far $\mathrm{CCl}$ have been already validated in patients with acute coronary syndrome ${ }^{27}$ and stroke $^{28}$ and other cardiovascular conditions ${ }^{9}$. Hence this study represents the first large evaluation of $\mathrm{CCl}$ in a population-based AF patients' cohort.

In recent years there has been an increasing need of new approaches to manage AF patients, considering them in a more comprehensive, integrated and holistic way. A systematic review and meta-analysis by Gallagher and colleagues showed how an integrated care approach can significantly reduce hospitalization and mortality in AF patients ${ }^{29}$. Various expert opinions and international consensus statements have proposed new integrated models to properly manage AF patients, with the ultimate objective to reduce the risk of adverse events ${ }^{30,31}$. The "Atrial Fibrillation Better Care" (ABC) pathway has recently been proposed as a possible model to integrate the various main aspects related to AF patients' management, in order to streamline and facilitate the integrated care and the holistic evaluation of these patients ${ }^{32}$ and showed to be associated with a significant lower risk of major adverse events ${ }^{33}$. Our data support this new approach and advocate the need for structured integrated and holistic management of AF patients. Adding a routine evaluation of the burden of multimorbidity in AF patients' clinical evaluation could help to identify those patients which would benefit more from integrated and holistic management, as described by the $A B C$ pathway, that could reduce the overall risk of major adverse events, beyond the "mere" baseline thromboembolic and bleeding risk.

\section{Limitations}

The main limitation of this study is related to the use of ICD-9 codes, that even if largely validated in clinical research, cannot completely exclude some risk of bias related to 
inaccuracies and coding mistakes; furthermore, this tool does not allow us to consider and evaluate some relevant factors for adverse outcomes in AF patients. Second, since all data are retrieved in the frame of hospital admissions, these results need to be cautiously generalized to the overall AF population. Nevertheless, administrative data are increasingly used in public health research because the information is readily available, collected in a standardized way, and inexpensive to use ${ }^{34}$. Third, using a relatively short period of time to build the clinical history and calculate $\mathrm{CCl}$ and not having available data before 2000 , some inaccuracy may lead to some underestimation of $\mathrm{CCl}$. Nevertheless, this inaccuracy would affect both the groups considered, hence with a minimal impact in terms of the overall results. Furthermore, we did not have any availability of data to calculate time in therapeutic range, a pivotal factor in determining major adverse events in AF patients treated with VKA ${ }^{35}$. Lastly, we used an adapted model of $\mathrm{CCl}$ conceived for the use in health administrative databases ${ }^{12}$. Despite this, currently the use of adapted $\mathrm{CCl}$ models to be used in ICD-9 codes-based databases is widely accepted ${ }^{36}$. Several adaptations have been proposed and have been considered similarly accurate and reliable in evaluating the burden of multimorbidity and risk of adverse events ${ }^{36}$. Notwithstanding these limitations, we provided the first evidence of a direct relationship between AF and increasing burden of multimorbidity and the largest validation of $\mathrm{CCl}$ as a reliable tool for evaluation of multimorbidity in AF patients and its association with major adverse events.

\section{CONCLUSIONS}

In hospitalized patients, $\mathrm{AF}$ is associated with an independent increase in $\mathrm{CCl}$, that was inversely associated with OAC prescriptions during follow-up. CCI was independently associated with an increased risk of stroke, major bleeding and all-cause death. New models of care able to consider the burden of comorbidities in AF patients and offer holistic approaches to AF management are needed. 


\section{REFERENCES}

1. Kirchhof P, Benussi S, Kotecha D, et al. 2016 ESC Guidelines for the management of atrial fibrillation developed in collaboration with EACTS. Eur Heart J. 2016;37(38):2893-2962. doi:10.1093/eurheartj/ehw210

2. Rahman F, Kwan GF, Benjamin EJ. Global epidemiology of atrial fibrillation. Nat Rev Cardiol. 2014;11(11):639-654. doi:10.1038/nrcardio.2014.118

3. Proietti M, Laroche $\mathrm{C}$, Nieuwlaat R, et al. Increased burden of comorbidities and risk of cardiovascular death in atrial fibrillation patients in Europe over ten years: A comparison between EORP-AF pilot and EHS-AF registries. Eur J Intern Med. 2018;55:28-34. doi:10.1016/j.ejim.2018.05.016

4. van den Akker M, Buntinx F, Metsemakers JF, Roos S, Knottnerus JA. Multimorbidity in general practice: prevalence, incidence, and determinants of cooccurring chronic and recurrent diseases. J Clin Epidemiol. 1998;51(5):367-375. http://www.ncbi.nlm.nih.gov/pubmed/9619963. Accessed July 19, 2018.

5. Barnett K, Mercer SW, Norbury M, Watt G, Wyke S, Guthrie B. Epidemiology of multimorbidity and implications for health care, research, and medical education: a cross-sectional study. Lancet. 2012;380(9836):37-43. doi:10.1016/S0140$6736(12) 60240-2$

6. Plsek PE, Greenhalgh T. Complexity science: The challenge of complexity in health care. BMJ. 2001;323(7313):625-628. doi:10.1136/bmj.323.7313.625

7. Forman DE, Maurer MS, Boyd C, et al. Multimorbidity in Older Adults With Cardiovascular Disease. J Am Coll Cardiol. 2018;71(19):2149-2161. doi:10.1016/j.jacc.2018.03.022

8. Charlson ME, Pompei P, Ales KL, MacKenzie CR. A new method of classifying prognostic comorbidity in longitudinal studies: development and validation. J Chronic Dis. 1987;40(5):373-383. http://www.ncbi.nlm.nih.gov/pubmed/3558716. Accessed 
July 18, 2018.

9. Rashid M, Kwok CS, Gale CP, et al. Impact of co-morbid burden on mortality in patients with coronary heart disease, heart failure, and cerebrovascular accident: a systematic review and meta-analysis. Eur Hear J - Qual Care Clin Outcomes. 2016;3(1):20-36. doi:10.1093/ehjqcco/qcw025

10. Lip GYH, Nieuwlaat R, Pisters R, Lane DA, Crijns HJGM. Refining clinical risk stratification for predicting stroke and thromboembolism in atrial fibrillation using a novel risk factor-based approach: the euro heart survey on atrial fibrillation. Chest. 2010;137(2):263-272. doi:10.1378/chest.09-1584

11. Lip GYH, Mauri L, Montalescot G, et al. Relationship of stroke and bleeding risk profiles to efficacy and safety of dabigatran dual therapy versus warfarin triple therapy in atrial fibrillation after percutaneous coronary intervention: An ancillary analysis from the RE-DUAL PCI trial. Am Heart J. 2019;212:13-22. doi:10.1016/j.ahj.2019.02.006

12. D'Hoore W, Bouckaert A, Tilquin C. Practical considerations on the use of the Charlson comorbidity index with administrative data bases. J Clin Epidemiol. 1996;49(12):1429-1433. http://www.ncbi.nIm.nih.gov/pubmed/8991959. Accessed March 17, 2017.

13. Boriani G, Proietti M. Atrial fibrillation prevention: an appraisal of current evidence. Heart. 2018;104(11):882-887. doi:10.1136/heartjnl-2017-311546

14. Gorenek B, Pelliccia A, Benjamin EJ, et al. European Heart Rhythm Association (EHRA)/European Association of Cardiovascular Prevention and Rehabilitation (EACPR) position paper on how to prevent atrial fibrillation endorsed by the Heart Rhythm Society (HRS) and Asia Pacific Heart Rhythm Society (AP. Europace. 2017;19(2):190-225. doi:10.1093/europace/euw242

15. Boriani G, Proietti M, Laroche C, et al. Contemporary stroke prevention strategies in 
11096 European patients with atrial fibrillation: A report from the EURObservational Research Programme on Atrial Fibrillation (EORP-AF) Long-Term General Registry. Europace. 2018;20(5):747-757. doi:10.1093/europace/eux301

16. Inohara T, Shrader P, Pieper K, et al. Association of of Atrial Fibrillation Clinical Phenotypes With Treatment Patterns and Outcomes: A Multicenter Registry Study. JAMA Cardiol. 2018;3(1):54-63. doi:10.1001/jamacardio.2017.4665

17. Vanbeselaere V, Truyers C, Elli S, et al. Association between atrial fibrillation, anticoagulation, risk of cerebrovascular events and multimorbidity in general practice: a registry-based study. BMC Cardiovasc Disord. 2016;16(1):61. doi:10.1186/s12872-016-0235-1

18. Jani BD, Nicholl BI, McQueenie R, et al. Multimorbidity and co-morbidity in atrial fibrillation and effects on survival: findings from UK Biobank cohort. Europace. November 2017. doi:10.1093/europace/eux322

19. Steinberg BA, Shrader P, Thomas L, et al. Factors associated with non-vitamin K antagonist oral anticoagulants for stroke prevention in patients with new-onset atrial fibrillation: Results from the Outcomes Registry for Better Informed Treatment of Atrial Fibrillation II (ORBIT-AF II). Am Heart J. 2017;189:40-47. doi:10.1016/j.ahj.2017.03.024

20. Marijon E, Le Heuzey J-Y, Connolly S, et al. Causes of death and influencing factors in patients with atrial fibrillation: a competing-risk analysis from the randomized evaluation of long-term anticoagulant therapy study. Circulation. 2013;128(20):21922201. doi:10.1161/CIRCULATIONAHA.112.000491

21. Fauchier L, Villejoubert O, Clementy N, et al. Causes of Death and Influencing Factors in Patients with Atrial Fibrillation. Am J Med. 2016;129(12):1278-1287. doi:10.1016/j.amjmed.2016.06.045

22. Pokorney SD, Piccini JP, Stevens SR, et al. Cause of Death and Predictors of All- 
Cause Mortality in Anticoagulated Patients With Nonvalvular Atrial Fibrillation: Data From ROCKET AF. J Am Heart Assoc. 2016;5(3). doi:10.1161/JAHA.115.002197

23. Proietti M, Laroche C, Opolski G, et al. "Real-world" atrial fibrillation management in Europe: observations from the 2-year follow-up of the EURObservational Research Programme-Atrial Fibrillation General Registry Pilot Phase. Europace. 2017;19(5):722-733. doi:10.1093/europace/euw112

24. Boriani G, Proietti M, Laroche $\mathrm{C}$, et al. Changes to oral anticoagulant therapy and risk of death over a 3-year follow-up of a contemporary cohort of European patients with atrial fibrillation final report of the EURObservational Research Programme on Atrial Fibrillation (EORP-AF) pilot general r. Int J Cardiol. 2018;271:68-74. doi:10.1016/j.ijcard.2018.05.034

25. Perera KS, Pearce LA, Sharma M, et al. Predictors of Mortality in Patients With Atrial Fibrillation (from the Atrial Fibrillation Clopidogrel Trial With Irbesartan for Prevention of Vascular Events [ACTIVE A]). Am J Cardiol. 2018;121(5):584-589.

doi:10.1016/j.amjcard.2017.11.028

26. Kim E-J, Yin X, Fontes JD, et al. Atrial fibrillation without comorbidities: Prevalence, incidence and prognosis (from the Framingham Heart Study). Am Heart J. 2016;177:138-144. doi:10.1016/j.ahj.2016.03.023

27. Radovanovic D, Seifert B, Urban P, et al. Validity of Charlson Comorbidity Index in patients hospitalised with acute coronary syndrome. Insights from the nationwide AMIS Plus registry 2002-2012. Heart. 2014;100(4):288-294. doi:10.1136/heartjnl2013-304588

28. Goldstein LB, Samsa GP, Matchar DB, Horner RD. Charlson Index comorbidity adjustment for ischemic stroke outcome studies. Stroke. 2004;35(8):1941-1945. doi:10.1161/01.STR.0000135225.80898.1c

29. Gallagher C, Elliott AD, Wong CX, et al. Integrated care in atrial fibrillation: A 
systematic review and meta-analysis. Heart. 2017;103(24):1947-1953.

doi:10.1136/heartjnl-2016-310952

30. Kirchhof $P$. The future of atrial fibrillation management: integrated care and stratified therapy. Lancet (London, England). 2017;390(10105):1873-1887.

doi:10.1016/S0140-6736(17)31072-3

31. Kotecha D, Breithardt G, Camm AJ, et al. Integrating new approaches to atrial fibrillation management: The 6th AFNET/EHRA Consensus Conference. Europace. 2018;20(3):395-407. doi:10.1093/europace/eux318

32. Lip GYH. The ABC pathway: an integrated approach to improve AF management. Nat Rev Cardiol. 2017;14(11):627-628. doi:10.1038/nrcardio.2017.153

33. Proietti M, Romiti GF, Olshansky B, Lane DA, Lip GYH. Improved Outcomes by Integrated Care of Anticoagulated Patients with Atrial Fibrillation Using the Simple ABC (Atrial Fibrillation Better Care) Pathway. Am J Med. August 2018. doi:10.1016/j.amjmed.2018.06.012

34. Rumsfeld JS, Joynt KE, Maddox TM. Big data analytics to improve cardiovascular care: promise and challenges. Nat Rev Cardiol. 2016;13(6):350-359. doi:10.1038/nrcardio.2016.42

35. Vestergaard AS, Skjøth F, Larsen TB, Ehlers LH. The importance of mean time in therapeutic range for complication rates in warfarin therapy of patients with atrial fibrillation: A systematic review and meta-regression analysis. Nagler M, ed. PLoS One. 2017;12(11):e0188482. doi:10.1371/journal.pone.0188482

36. de Groot V, Beckerman H, Lankhorst GJ, Bouter LM. How to measure comorbidity. a critical review of available methods. J Clin Epidemiol. 2003;56(3):221-229. http://www.ncbi.nlm.nih.gov/pubmed/12725876. Accessed May 8, 2019. 


\section{FIGURES LEGENDS}

Figure 1: Charlson Comorbidity Index Trends according to Atrial Fibrillation Diagnosis

Legend: Whiskers stand for standard deviation of mean; $\mathrm{AF}=$ Atrial Fibrillation; $\mathrm{CCl}=$ Charlson Comorbidity Index.

Figure 2: Kaplan-Meier Curves for Major Adverse Events according to Charlson Comorbidity Index Classes

Legend: $\mathrm{CCl}=$ Charlson Comorbidity Index. 
Table 1: Baseline Characteristics according to Atrial Fibrillation and Charlson Comorbidity Index

\begin{tabular}{|c|c|c|c|c|c|c|}
\hline & \multirow{3}{*}{$\begin{array}{c}\text { Non-AF } \\
N=240400\end{array}$} & \multirow{3}{*}{$\begin{array}{c}\text { AF } \\
N=24040\end{array}$} & \multirow[t]{3}{*}{$\mathbf{P}$} & \multicolumn{3}{|c|}{ AF } \\
\hline & & & & $\mathrm{CCl} \mathrm{0-3}$ & $\mathrm{CCl} \geq 4$ & $\mathbf{P}$ \\
\hline & & & & $N=19745$ & $N=4295$ & \\
\hline Age, years mean $\pm \mathrm{SD}$ & $59.7 \pm 13.2$ & $76.1 \pm 9.8$ & $<.001$ & $75.7 \pm 9.9$ & $77.8 \pm 8.8$ & $<.001$ \\
\hline Age classes, n (\%) & & & $<.001$ & & & $<.001$ \\
\hline$<65$ years & $155310(64.6)$ & $2964(12.3)$ & & $2651(13.4)$ & $313(7.3)$ & \\
\hline $65-74$ years & $47525(19.8)$ & $6702(27.9)$ & & $5611(28.4)$ & $1091(25.4)$ & \\
\hline$\geq 75$ years & $37565(15.6)$ & $14374(59.8)$ & & $11483(58.2)$ & $2891(67.3)$ & \\
\hline Male, $\mathrm{n}(\%)$ & $11096(46.2)$ & $12079(50.2)$ & $<.001$ & $9841(49.8)$ & $2238(52.1)$ & $<.001$ \\
\hline Charlson Comorbidity Index, $($ mean $\pm S D)$ & $0.2 \pm 0.9$ & $1.8 \pm 2.1$ & $<.001$ & $1.1 \pm 1.1$ & $5.5 \pm 1.8$ & $<.001$ \\
\hline Hypertension, n (\%) & $79801(33.2)$ & $18605(77.4)$ & $<.001$ & $15452(78.3)$ & $3153(73.4)$ & $<.001$ \\
\hline Diabetes Mellitus, n (\%) & $4316(1.8)$ & $3555(14.8)$ & $<.001$ & $1763(8.9)$ & $1792(41.7)$ & $<.001$ \\
\hline Myocardial Infarction, n (\%) & $1723(0.7)$ & $1400(5.8)$ & $<.001$ & $869(4.4)$ & $531(12.4)$ & $<.001$ \\
\hline Congestive Heart Failure, $\mathrm{n}(\%)$ & $2919(1.2)$ & $7249(30.1)$ & $<.001$ & $4882(24.7)$ & $2367(55.1)$ & $<.001$ \\
\hline Cerebrovascular Disease, $\mathrm{n}(\%)$ & $3216(1.3)$ & $3605(15.0)$ & $<.001$ & $1625(8.2)$ & $1980(46.1)$ & $<.001$ \\
\hline Hemiplegia, $n(\%)$ & $2282(0.9)$ & $2830(11.8)$ & $<.001$ & $1027(5.2)$ & $1803(42.0)$ & $<.001$ \\
\hline Dementia, n (\%) & $489(0.2)$ & $400(1.7)$ & $<.001$ & $197(1.0)$ & $203(4.7)$ & $<.001$ \\
\hline COPD, n (\%) & $3125(1.3)$ & $4017(16.7)$ & $<.001$ & $2523(12.8)$ & $1494(34.8)$ & $<.001$ \\
\hline Connective Tissue Disease, $\mathrm{n}(\%)$ & $560(0.2)$ & $303(1.3)$ & $<.001$ & $228(1.1)$ & $75(1.7)$ & .002 \\
\hline Ulcer, n (\%) & $620(0.3)$ & $440(1.8)$ & $<.001$ & $287(1.4)$ & $153(3.6)$ & $<.001$ \\
\hline Mild Liver Disease, $\mathrm{n}(\%)$ & $1918(0.8)$ & $1212(5.0)$ & $<.001$ & $669(3.4)$ & $543(12.6)$ & $<.001$ \\
\hline Moderate/Severe Liver disease, $n(\%)$ & $769(0.3)$ & $334(1.4)$ & $<.001$ & $44(0.2)$ & $290(6.7)$ & $<.001$ \\
\hline
\end{tabular}




\begin{tabular}{|c|c|c|c|c|c|c|}
\hline Renal Disease, $n(\%)$ & $1244(0.5)$ & $2087(8.7)$ & $<.001$ & $788(4.0)$ & $1299(30.24)$ & $<.001$ \\
\hline Metastatic Tumor, $\mathrm{n}(\%)$ & $1162(0.5)$ & $503(2.1)$ & $<.001$ & $0(0.0)$ & $503(11.7)$ & $<.001$ \\
\hline Leukemia, $\mathrm{n}(\%)$ & $117(0.1)$ & $86(0.4)$ & $<.001$ & $49(0.2)$ & $37(0.9)$ & $<.001$ \\
\hline Lymphoma, n (\%) & $305(0.1)$ & $190(0.8)$ & $<.001$ & $90(0.5)$ & $100(2.3)$ & $<.001$ \\
\hline Any Tumor, n (\%) & $4423(1.8)$ & $2189(9.1)$ & $<.001$ & $1124(5.7)$ & $1065(24.8)$ & $<.001$ \\
\hline $\mathrm{CHA}_{2} \mathrm{DS}_{2}$-VASc, $($ mean $\pm S D)$ & $1.4 \pm 1.2$ & $3.3 \pm 1.4$ & $<.001$ & $3.2 \pm 1.3$ & $4.1 \pm 1.5$ & $<.001$ \\
\hline mHAS-BLED, (mean $\pm S D)$ & $0.9 \pm 1.0$ & $2.3 \pm 1.0$ & $<.001$ & $2.1 \pm 0.9$ & $2.8 \pm 1.1$ & $<.001$ \\
\hline Oral Anticoagulant Drugs, $\mathrm{n}(\%)$ & $4141(1.7)$ & $9646(40.1)$ & $<.001$ & $8358(42.3)$ & $1288(30.0)$ & $<.001$ \\
\hline
\end{tabular}

Legend: $\mathrm{AF}=$ Atrial Fibrillation; $\mathrm{CCl}=$ Charlson Comorbidity Index; COPD= Chronic Obstructive Pulmonary Disease; SD= Standard

Deviation. 
Table 2: Logistic Regression Analysis for Oral Anticoagulant Drugs Prescription according to Charlson Comorbidity Index

\begin{tabular}{|c|c|c|c|c|c|c|}
\hline & \multicolumn{3}{|c|}{ OAC Prescription at Baseline } & \multicolumn{3}{|c|}{ OAC Prescription at End of Follow-Up } \\
\hline & OR & $95 \% \mathrm{Cl}$ & $\mathbf{P}$ & OR & $95 \% \mathrm{Cl}$ & $\mathbf{P}$ \\
\hline $\mathbf{C C l}$ (as continuous variable) & 0.91 & $0.89-0.92$ & $<.001$ & 0.98 & $0.98-0.99$ & .03 \\
\hline \multirow[t]{3}{*}{$\mathrm{CCI} \geq \mathbf{4}$ (vs. CCI 0-3) } & 0.65 & $0.60-0.70$ & $<.001$ & 0.98 & $0.93-1.04$ & .50 \\
\hline & \multicolumn{3}{|c|}{ VKA Prescription at End of Follow-Up } & \multicolumn{3}{|c|}{ NOACs Prescription at End of Follow-Up } \\
\hline & OR & $95 \% \mathrm{Cl}$ & $\mathbf{P}$ & OR & $95 \% \mathrm{Cl}$ & $\mathbf{P}$ \\
\hline $\mathbf{C C I}$ (as continuous variable) & 0.99 & $0.98-1.00$ & .22 & 0.86 & $0.81-0.90$ & $<.001$ \\
\hline $\mathrm{CCl} \geq 4$ (vs. CCl 0-3) & 1.00 & $0.95-1.06$ & .94 & 0.48 & $0.37-0.63$ & $<.001$ \\
\hline
\end{tabular}

Legend: $\mathrm{CCl}=$ Charlson Comorbidity Index; NOACs= Non-Vitamin K Antagonists Oral Anticoagulants; OAC= Oral Anticoagulants;

$\mathrm{VKA}=$ Vitamin $\mathrm{K}$ Antagonist. 
Table 3: Cox Regression Analysis for Major Adverse Events

\begin{tabular}{|c|c|c|c|c|c|c|}
\hline & \multicolumn{6}{|c|}{$\begin{array}{l}\text { Charlson Comorbidity Index } \\
\text { (as continuous time-dependent variable) }\end{array}$} \\
\hline & \multicolumn{3}{|c|}{ Model $1^{*}$} & \multicolumn{3}{|c|}{ Model 2† } \\
\hline & HR & $95 \% \mathrm{Cl}$ & $\mathbf{P}$ & HR & $95 \% \mathrm{Cl}$ & $\mathbf{P}$ \\
\hline Stroke & 1.04 & $1.03-1.06$ & $<.001$ & 1.04 & $1.02-1.06$ & $<.001$ \\
\hline Major Bleeding & 1.02 & $0.99-1.04$ & .15 & 1.03 & $1.01-1.06$ & $<.001$ \\
\hline All-Cause Death & 1.10 & $1.09-1.11$ & $<.001$ & 1.10 & $1.09-1.11$ & $<.001$ \\
\hline
\end{tabular}

Legend: *adjusted for sex and age; †adjusted for sex, age and use of OAC; Cl= Confidence Interval; HR= Hazard Ratio; OAC= Oral

Anticoagulant. 


\section{Long-Term Relationship between Atrial Fibrillation, Multimorbidity}

and Oral Anticoagulant Drugs Use

Marco Proietti, Irene Marzona, Tommaso Vannini, Mauro Tettamanti, Ida Fortino, Luca Merlino, Stefania Basili, Pier Mannuccio Mannucci, Giuseppe Boriani, Gregory YH Lip, Maria Carla Roncaglioni, Alessandro Nobili

Supplementary Materials 
eTable 1: ICD-9 Codes

\begin{tabular}{|c|c|c|}
\hline Condition & ICD-9/ATC Codes & Weight \\
\hline Myocardial Infarction & 410.xx, 411.xx & 1 \\
\hline Congestive Heart Failure & 398.xx, 402.xx, 428.xx & 1 \\
\hline Peripheral Vascular Disease & 440.xx-447.xx & 1 \\
\hline Dementia & 290.xx, 291.xx, 294.xx & 1 \\
\hline Cerebrovascular Disease & 430.xx-433.xx, 435.xx & 1 \\
\hline COPD & 491.xx-493.xx & 1 \\
\hline Connective Tissue Disease & 710.xx, 714.xx, 725.xx & 1 \\
\hline Ulcer Disease & 531.xx-534.xx & 1 \\
\hline Mild Liver Disease & 571.xx, 573.xx & 1 \\
\hline Hemiplegia & $\begin{array}{c}\text { 342.xx, 434.xx, 436.xx } \\
\text { 437.xx }\end{array}$ & 2 \\
\hline $\begin{array}{c}\text { Moderate or Severe Renal } \\
\text { Disease }\end{array}$ & $\begin{array}{c}\text { 403.xx, 404.xx, 580.xx- } \\
\text { 586.xx }\end{array}$ & 2 \\
\hline Diabetes Mellitus & $250 . x x$ & 2 \\
\hline Any Tumor & 140.xx-195.xx & 2 \\
\hline Leukemia & 204.xx-208.xx & 2 \\
\hline Lymphoma & 200.xx, 202.xx, 203.xx & 2 \\
\hline $\begin{array}{c}\text { Moderate or Severe Liver } \\
\text { Disease }\end{array}$ & 070.xx, 570.xx, 572.xx & 3 \\
\hline Metastatic Solid Tumor & 196.xx-199.xx & 6 \\
\hline Hypertension & $\begin{array}{c}\text { ATC: C02, C03, C07, } \\
\text { C08, C09 }\end{array}$ & - \\
\hline
\end{tabular}




\begin{tabular}{|c|c|c|}
\hline Stroke & 434.xx, 436.xx & - \\
\hline Major Bleeding & 430.xx, 431.xx, 432.xx, & - \\
& $459.0,530.82,569.3$, & \\
& $578 . x x$ & \\
\hline
\end{tabular}

Legend: $\mathrm{ATC}=$ Anatomical Therapeutic Classification; COPD= Chronic Obstructive Pulmonary Disease; $I C D=$ International Classification of Disease. 
eTable 2: Charlson Comorbidity Index

\begin{tabular}{|c|c|}
\hline Condition & Weight \\
\hline Myocardial Infarction & 1 \\
\hline Congestive Heart Failure & 1 \\
\hline Peripheral Vascular Disease & 1 \\
\hline Cerebrovascular Disease & 1 \\
\hline Dementia & 1 \\
\hline COPD & 1 \\
\hline Connective Tissue Disease & 1 \\
\hline Ulcer Disease & 1 \\
\hline Mild Liver Disease & 1 \\
\hline Diabetes Mellitus & 1 \\
\hline Hemiplegia & 2 \\
\hline Moderate or Severe Renal Disease & 2 \\
\hline $\begin{array}{c}\text { Diabetes Mellitus with End-Organ } \\
\text { Damage }\end{array}$ & 2 \\
\hline Any Tumor & 2 \\
\hline Leukemia & 2 \\
\hline Lymphoma & 2 \\
\hline Moderate or Severe Liver Disease & 3 \\
\hline Autoimmune Deficiency Syndrome & 6 \\
\hline Metastatic Solid Tumor & 6 \\
\hline
\end{tabular}

Legend: COPD= Chronic Obstructive Pulmonary Disease. 
eTable 3: Major Adverse Events according to Charlson Comorbidity Index Classes

\section{CCl 0-3}

$\mathrm{N}=19745$

$\mathrm{CCl} \geq 4$

\begin{tabular}{lccrr} 
& \multicolumn{2}{c}{$\mathrm{N}=19745$} & \multicolumn{2}{c}{$\mathrm{N}=4295$} \\
\cline { 2 - 5 } & $\mathrm{N}$ & Cumulative Incidence $^{*}$ & $\mathrm{~N}$ & Cumulative Incidence $^{*}$ \\
\hline Stroke & 1826 & 17.4 & 412 & 26.0 \\
Major Bleeding & 1120 & 12.0 & 197 & 15.7 \\
All-Cause Death & 13831 & 76.0 & 3650 & 95.0
\end{tabular}

Legend: *per 100 patients; $\mathrm{CCl}=$ Charlson Comorbidity Index. 
eFigure 1: Charlson Comorbidity Index Trends according to Atrial Fibrillation Diagnosis in Patients $<65$ years old

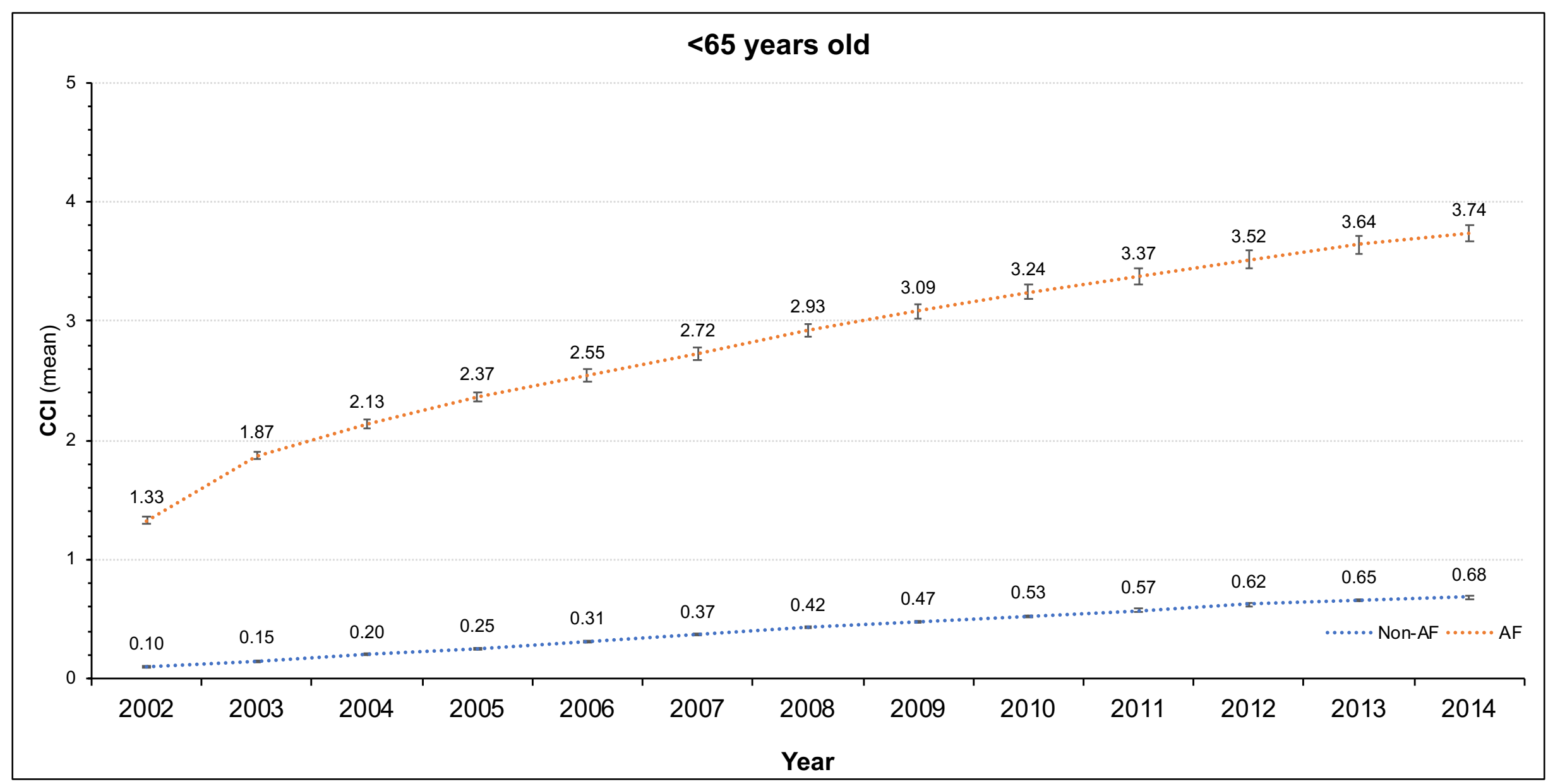

Legend: Whiskers stand for standard deviation of mean; $\mathrm{AF}=$ Atrial Fibrillation; $\mathrm{CCl}=$ Charlson Comorbidity Index. 
eFigure 2: Charlson Comorbidity Index Trends according to Atrial Fibrillation Diagnosis in Patients 65-74 years old

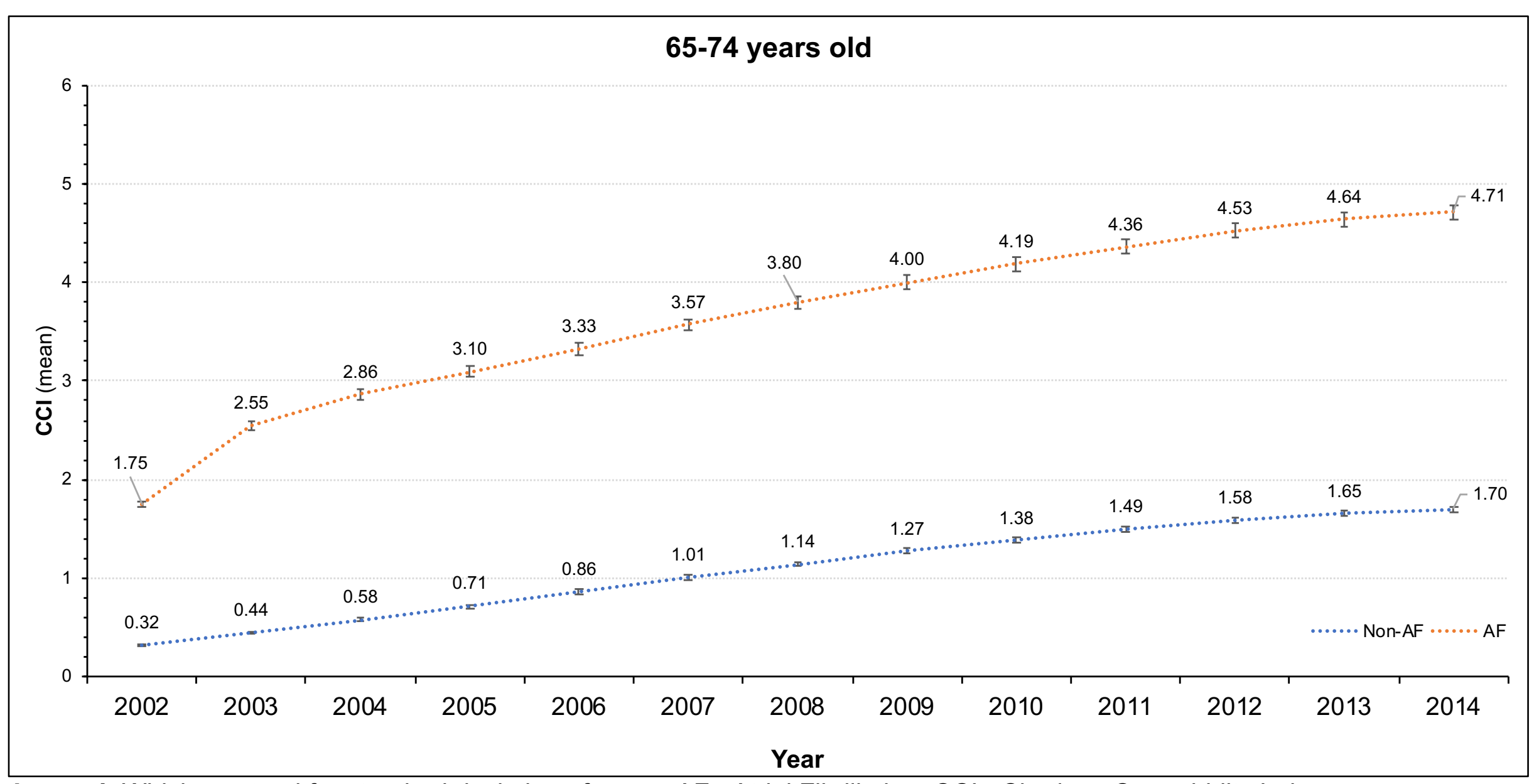

Legend: Whiskers stand for standard deviation of mean; $\mathrm{AF}=$ Atrial Fibrillation; $\mathrm{CCl}=$ Charlson Comorbidity Index. 
eFigure 3: Charlson Comorbidity Index Trends according to Atrial Fibrillation Diagnosis in Patients $\geq 75$ years old

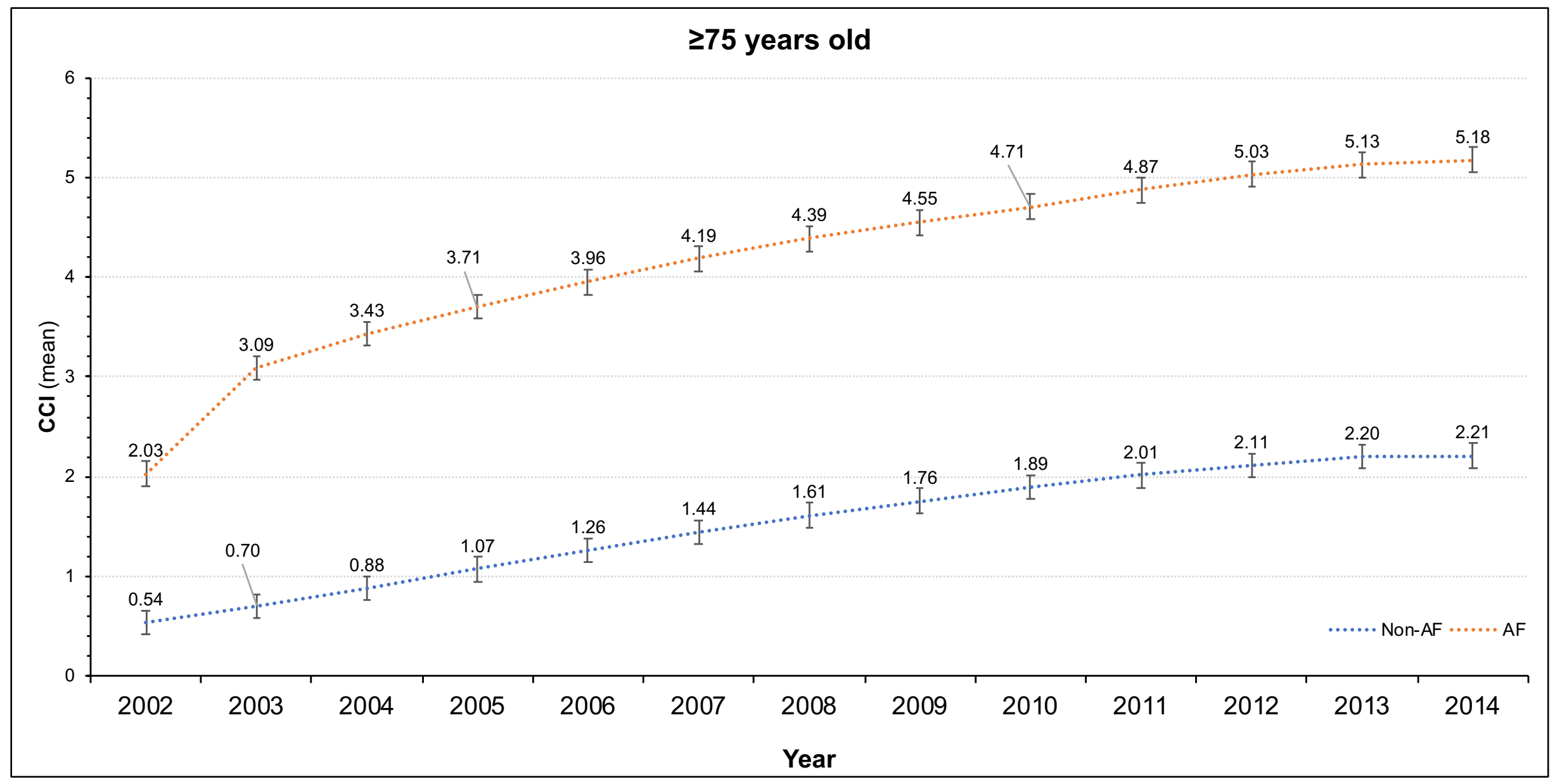

Legend: Whiskers stand for standard deviation of mean; AF= Atrial Fibrillation; $\mathrm{CCl}=$ Charlson Comorbidity Index. 